\title{
Functional Decomposition in 5G Networks
}

\author{
Davit Harutyunyan ${ }^{(凶)}$ and Roberto Riggio \\ CREATE-NET, via Alla Cascata 56/C, Trento, Italy \\ \{dharutyunyan,rriggio\}@create-net.org
}

\begin{abstract}
Mobile data traffic has been rapidly increasing over the last few years. To accommodate for such an ever-growing traffic demand, mobile network operators are required to perform costly network upgrades. Cell size reduction and network virtualization are known to be two of the most effective ways to increase capacity and lower the cost to deploy and operate future mobile networks. This $\mathrm{PhD}$ thesis will study the trade-offs associated with the different approaches to small cell virtualization in terms of the point at which base stations operations are decomposed into physical and virtual. Different functional splits will be compared using mathematical optimization tools (e.g., Integer Linear Programming) while novel heuristics will be devised in order to tackle problems of practical size. Finally, selected results will be empirically evaluated over the 5G-EmPOWER testbed deployed at CREATE-NET premises.
\end{abstract}

Keywords: Mobile networks $\cdot$ Functional split $\cdot$ Component placement

\section{Introduction}

Recent advances in network virtualization enabled mobile network operators (MNOs) to move from the D-RAN (Distributed Radio Access Network) architecture, where base-band processing and radio elements are co-located, to the C-RAN (Cloud-RAN) architecture, where baseband units are decomposed from the radio elements and are consolidated in large data-centers. The vaunted benefits of $\mathrm{C}-\mathrm{RAN}$ are enhanced radio resource utilization and coordination across multiple cells enabled by the centralization of the radio resource management tasks. The downside of such high level of centralization lies in the tight bandwidth and latency requirements imposed on the fronthaul, i.e. the link interconnecting RRHs (Remote Radio Heads) with the BBUs (Base-Band Units), which can usually be satisfied only using fiber links.

Nevertheless, although the link between RRHs and BBU Pool has been used as demarcation point in the $\mathrm{C}-\mathrm{RAN}$ architecture, other functional splits can be in principle defined (see Fig. 1), each of them coming with different requirements. In general, the lower the functional split is executed within the RAN protocol stack, the higher is the centralization benefits, however the fronthaul requirements become also more stringent. For example C-RAN enables an MNOs to implement Coordinated Multi-Point (CoMP) transmission and reception, while 


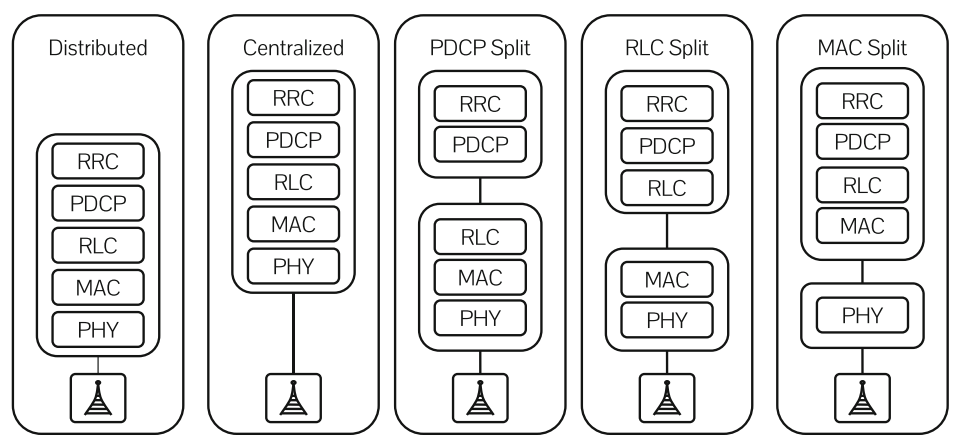

Fig. 1. Different functional splits for 5G small-cells [1].

a split above the Medium Access Control (MAC) layer cannot support CoMP only allowing higher-layers cooperation features (e.g., joint scheduling).

This $\mathrm{PhD}$ thesis will explore the trade-offs associated with the different functional splits, including, fronthaul requirements, centralization benefits, and deployment costs, with the ultimate goal of providing MNOs with a set guidelines to design cost-efficient mobile networks. In this paper we report on some preliminary results on the BBU placement problem over a reconfigurable wireless fronthaul while also presenting a roadmap for this $\mathrm{PhD}$ thesis.

\section{Background}

A detailed discussion on various functional splits can be found in [2-4]. The authors of [2] propose a novel RAN as a Service concept in which the level of centralization is flexible and can be adapted to the actual service demands. Several functional splits are introduced and the associated fronthaul requirements are provided in [3]. The authors of [4] survey several wired/wireless fronthauling technologies as well as the associated bandwidth and latency requirements for different functional splits.

Extensive work on $\mathrm{C}-\mathrm{RAN}$ has been published in the recent years [5-8]. In [5] the authors propose the Colony-RAN architecture for cellular systems able to adjust the cell layout by dynamically changing the connections between BBUs and RRHs. An ILP problem is formalized in [7] for optimizing the assignment of cells to different BBU pools. The authors of [8] derive a mathematical model for optimizing $\mathrm{C}-\mathrm{RAN}$ deployments combining fiber and microwave links.

The amount of literature on VNE (Virtual Network Embedding) is humbling, a comprehensive survey on this topic can be found in [9]. Likewise, also the amount of work on VNF (Virtual Network Function) placement is considerable [9-13]. In [14] SiMPLE is proposed to address the survivable VNE problem, exploiting a physical path diversity to provide survivability against single and multiple path failures. Similarly, the authors of [15] put forward a batch approach for survivable VNE. Joint node and link embedding algorithms are 
presented in $[16,17]$. A VNF placement problem is proposed in [10] for the radio access network, while a dynamic VNF placement problem is presented in [11]. The authors of [12] consider a hybrid environment in which part of the services are provided by dedicated hardware. In [13] an online VNF scheduling and placement problem is formulated.

\section{Research Statement}

Figure 2 depicts the reference network architecture envisioned in this $\mathrm{PhD}$ thesis. In the lower part of the figure we can see a traditional C-RAN deployment where all the BBUs are centralized and long fiber links are used in order to connect BBUs with RRHs (solid black lines). In the upper part of the figure instead, we can see the architecture envisioned in this work. In this case BBU Pools are co-located with macro cells and a reconfigurable wireless fronthaul is used to connect BBUs with RRHs (dashed black lines). A traditional backhaul is used in order to connect macro cells to the core network. This approach has the potential to reduce the length of the (expensive) fiber fronthaul links, thus improving operational and capital expenses, while still enabling advanced control and coordination mechanisms.

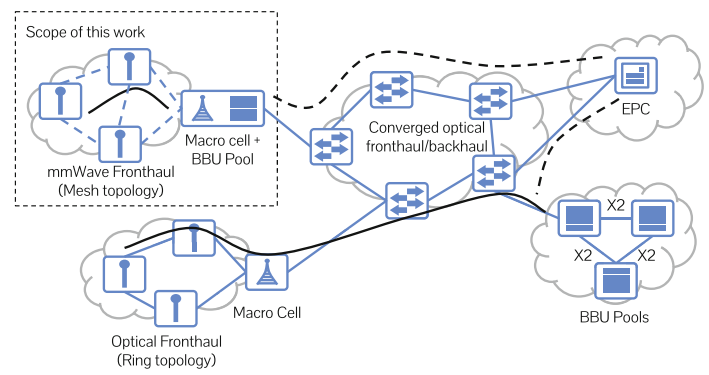

Fig. 2. The reference network architecture.

This architecture will be the starting point for this $\mathrm{PhD}$ thesis and will be extended in order to accommodate for different functional splits and for different fronhauling technologies. For each functional split we will formulate an optimization problem and we will study the associated trade-offs. The key performance indicators will include energy consumption as well as network performance (throughput, latency) and resiliency. Scalable heuristics will be developed in order to study networks of practical sizes. We will compare the performances of the ILP-based placement algorithm and of the heuristics using a custom discrete event simulator implemented in Matlab and based on the results we will implement a particular split in the 5G-EmPOWER testbed deployed at CREATE-NET premises ${ }^{1}$. Finally, we will address the economical implications

\footnotetext{
${ }^{1}$ http://empower.create-net.org/.
} 
of the different splits with the overarching objective of providing MNOs a set of guidelines for designing and deploying future $5 \mathrm{G}$ networks.

\section{Preliminary Result}

As preliminary work we formalized and solved the BBU Placement problem over a reconfigurable wireless fronthaul. This approach is made possible by recent advances in microwave communications which allow for up to a few Gbps of bandwidth over short distances, (i.e., less than one $\mathrm{Km}$ ). In the BBU placement problem the input consists of virtual network requests composed by a variable number of small cells and BBUs, whereas the substrate network provides the physical constraints in terms of bandwidth and computational capacity. In the evaluation we considered grid-shaped substrate networks and starshaped virtual network requests. We formulate the BBU placement problem as an ILP problem and we propose a greedy heuristic. The ILP problem formulation, including the objective function and the associated constraints as well as the details of the greedy heuristic, have been omitted due to space limitations. Simulations are carried out in Matlab. In this study we assume that a fixed number of requests are embedded sequentially onto the substrate network. Figure 3 shows the percentage of accepted requests and the average embedding cost for different substrate networks. As expected the ILP-based placement algorithm is more efficient than the heuristic in mapping the incoming requests. Nevertheless the performance gap, in this particular case, is relatively small while, as expected, the time required to embed a single request using the greedy heuristic is roughly one order of magnitude smaller than the time required to perform the same embedding using the ILP-based algorithm.

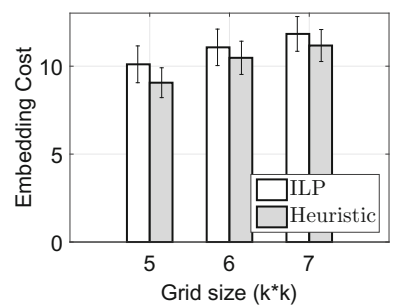

(a) Embedding Cost.

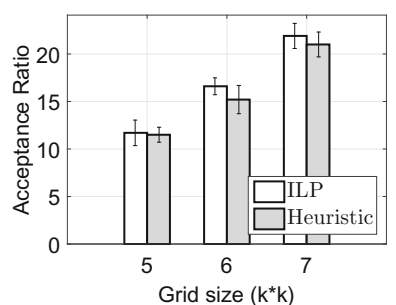

(b) Acceptance Ratio.

Fig. 3. ILP-based algorithm and heuristics performance for different networks.

\section{Conclusion}

In this paper we presented a roadmap for a comprehensive study on the tradeoffs associated with different approaches to small cell virtualization in $5 \mathrm{G}$ mobile networks. The study will account for a broad range of key performance indicators 
ranging from energy consumption to deployment costs and will produce major results in terms of design guidelines and resource allocation algorithms for future mobile networks. Selected results will also be empirically evaluated over the $5 \mathrm{G}-$ EmPOWER testbed deployed at CREATE-NET premises.

\section{References}

1. Small cell virtualization functional splits and use cases. Small Cell Forum, Technical report, June 2015

2. Rost, P., Bernardos, C., Domenico, A., Girolamo, M., Lalam, M., Maeder, A., Sabella, D., et al.: Cloud technologies for flexible $5 \mathrm{G}$ radio access networks. IEEE Commun. Mag. 52(5), 68-76 (2014)

3. Wubben, D., Rost, P., Bartelt, J.S., Lalam, M., Savin, V., Gorgoglione, M., Dekorsy, A., Fettweis, G.: Benefits and impact of cloud computing on $5 \mathrm{G}$ signal processing: flexible centralization through cloud-ran. IEEE Sig. Process. Mag. 31(6), 35-44 (2014)

4. Maeder, A., Lalam, M., De Domenico, A., Pateromichelakis, E., Wubben, D., Bartelt, J., Fritzsche, R., Rost, P.: Towards a flexible functional split for cloudran networks. In: Proceedings of EuCNC, Bologna (2014)

5. Namba, S., Matsunaka, T., Warabino, T., Kaneko, S., Kishi, Y.: Colony-RAN architecture for future cellular network. In: Proceedings of IEEE FutureNetw, Berlin (2012)

6. Carapellese, N., Tornatore, M., Pattavina, A.: Placement of base-band units (BBUs) over fixed/mobile converged multi-stage WDM-PONs. In: Proceedings of IEEE ONDM, Brest (2013)

7. Holm, H., Checko, A., Al-obaidi, R., Christiansen, H.: Optimal assignment of cells in C-RAN deployments with multiple BBU pools. In: Proceedings of EuCNC, Paris (2015)

8. Al-obaidi, R., Checko, A., Holm, H., Christiansen, H.: Optimizing Cloud-RAN deployments in real-life scenarios using microwave radio. In: Proceedings of EuCNC, Paris (2015)

9. Fischer, A., Botero, J.F., Till Beck, M., De Meer, H., Hesselbach, X.: Virtual network embedding: a survey. IEEE Commun. Surv. Tutorials 15(4), 1888-1906 (2013)

10. Riggio, R., Rasheed, T., Narayanan, R.: Virtual network functions orchestration in enterprise WLANs. In: Proceedings of IEEE ManFI, Ottawa (2015)

11. Clayman, S., Maini, E., Galis, A., Manzalini, A., Mazzocca, N.: The dynamic placement of virtual network functions. In: Proceedings of IEEE NOMS, Krakow (2014)

12. Moens, H., De Turck, F.: VNF-P: a model for efficient placement of virtualized network functions. In: Proceedings of IEEE CNSM, Rio de Janeiro (2014)

13. Mijumbi, R., Serrat, J., Gorricho, J.-L., Bouten, N., De Turck, F., Davy, S.: Design and evaluation of algorithms for mapping and scheduling of virtual network functions. In: Proceedings of IEEE NetSoft, London (2015)

14. Khan, M.M.A., Shahriar, N., Ahmed, R., Boutaba, R.: SiMPLE: survivability in multi-path link embedding. In: Proceedings of IEEE CNSM, Barcelona (2015)

15. Soualah, O., Fajjari, I., Aitsaadi, N., Mellouk, A.: A batch approach for a survivable virtual network embedding based on Monte-Carlo tree search. In: Proceedings of IEEE IM, Ottawa (2015) 
16. Despotovic, Z., Hecker, A., Malik, A.N., Guerzoni, R., Vaishnavi, I., Trivisonno, R., Beker, S.A.: VNetMapper: a fast and scalable approach to virtual networks embedding. In: Proceedings of IEEE ICCCN, Shanghai (2014)

17. Guerzoni, R., Trivisonno, R., Vaishnavi, I., Despotovic, Z., Hecker, A., Beker, S., Soldani, D.: A novel approach to virtual networks embedding for SDN management and orchestration. In: Proceedings of IEEE NOMS, Krakow (2014) 